\title{
Draft Design of Over-The-Air of Internet of Things for Farming
}

\author{
ByungRae Cha, Sun Park, JongWon Kim, and Byeong-Chun Shin
}

\begin{abstract}
Over the next 20 years it will begin the exodus from the Internet and smart phones to the Internet of Things. The heart of IoT gives new utility and value with connectivity among things around people to the human. The proposed IoT or IIoT based Download OTA (Over-the-Air) provides a flexible mechanism for downloading Media objects of any type and size from a network. Moreover, proposed IoT based DLOTA provides a part of security by CapBAC technique.

Keywords- IoF(IoT for Farming); IoF-Cloud; OTA; CapBAC
\end{abstract}

\section{Introduction}

Since the desktop was connected to the Internet, it took about 20 years for the Internet to connect to the smartphone. The era of the Internet of things that has been unfamiliar since the last few years has come close to us, over the next 20 years it will begin the exodus from the Internet and smart phones to the Internet of Things. The heart of IoT gives new utility and value with connectivity among things around people to the human. Recently, In addition to Google, the companies such as Samsung and Intel are jumping into the Internet market, which is probably the answer to the slowing growth of the smartphone market. If the smartphone is made to be able to be connected to anytime and anywhere around the human being, the Internet will be able to connect all things around people and make them communicate with each other. The Internet of Things is expected to lead the next smartphone market and the new blue ocean on behalf of smartphones that have entered a period of stagnant growth [1]. In addition, the future industrial environment will be driven by various changes centering on Industry 4.0, and all processes including product, machine, plant and factory will be closely connected through leap to industry 4.0. The core concept of Industry 4.0 is digitalization, and market trends are toward modularization, digital drawing at the production stage, and communication between machines. By finally digitizing the entire product and production cycle, a combination of the virtual world and the real world, the digital factory will finally become a reality [2].

Industrial IoT (IIoT) is growing in importance due to the expansion of the elements that make up the ecosystem and various devices.

ByungRae Cha, Sun Park, and JongWon Kim

School of Electrical Engineering and Computer Science, GIST, Korea \{brcha, sunpark, jongwon\}

\section{Byeong-Chun Shin}

Dept. of Mathematics, Chonnam Univ., Korea bcshin

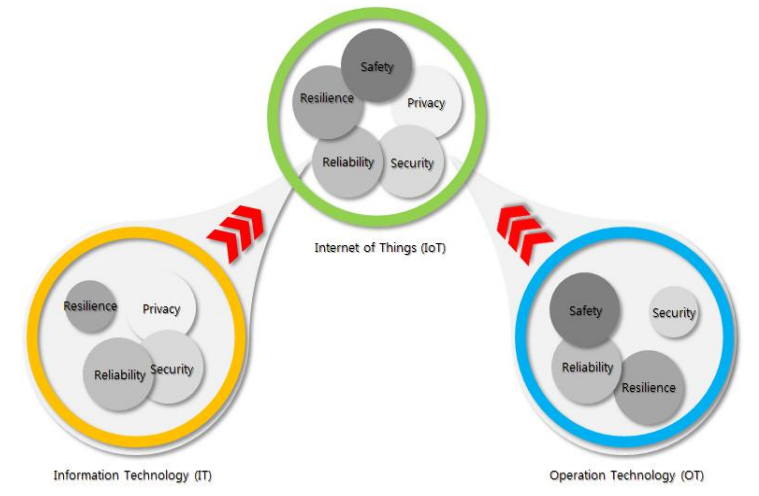

Figure 1. Convergence of Information Technology and Operation Technology

IIoT security can be secured by the fusion of information technology and operational technology, and reliability of information technology is security, reliability, privacy, and resilience aspect. Operational technology reliability is safety, resilience, reliability and security as shown in Fig. 1. [3]. In related work of this paper, OMA's OTA, SOTA / FOTA, and IoF-Cloud are briefly described. In Section 3, IoT devicebased DLOTA mechanism of IoF-Cloud is proposed. Finally, the conclusions and future studies are described.

\section{Related Work}

\section{A. OMA's Download OTA}

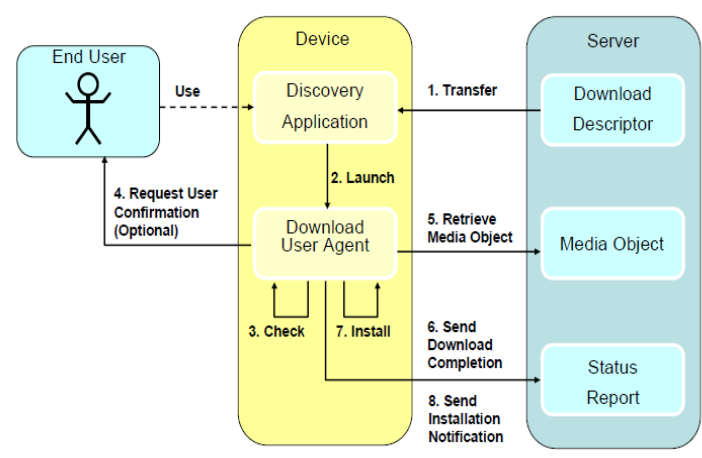

Figure 2. OMA's Dwonload Mechanism

The Open Mobile Alliance (OMA)'s Over-the-Air (OMA) provides a flexible mechanism for downloading media objects (MOs) of any format and size over the network [4, 5]. Fig. 2 shows the downloading process of OMA. Recently, OTA technology is divided into SOTA (Software Over-the-Air) and 
FOTA (Firmware Over-the-Air) in the field of Connected Care, and Fig. 3 shows the comparison of FOTA / SOTA technologies in mobile and automobile fields [6].
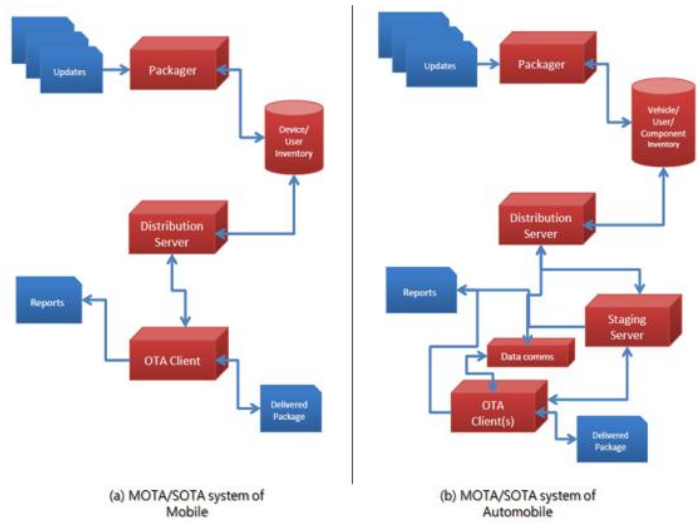

Figure 3. Comparation of OTA Technology in Mobile and Automobile fields

\section{B. Capability-based Access Control ( $\mathrm{CapBAC)}$}

Capability-based access control is that the subject has an authority list and presents its own capability to the service provider. The service provider confirms and approves the capability. In the case of repetitive service requests, the ACLbased system processes the authentication process repeatedly, but Capability-based access control can minimize the repetitive tasks through the published capability, and thus the workflow is light compared to the ACL-based system as shown in Fig. 4 [7-9].
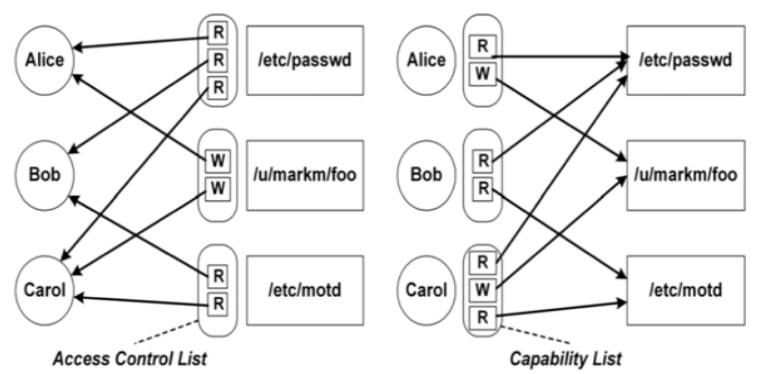

Figure 4. Concept Comparasion ACL and CapBAC

\section{Concept of IoF-Cloud}

IoT (Internet of Things) is a concept that creates new value when objects are connected to each other. In the dictionary meaning of things, it includes not only material objects but also intangible things [10]. It is changing and evolving into things that include things from types to intangible services. IoF (Internet of Farming)-Cloud is defined to extend this concept to farmers. IoF-Cloud means extending smart farms based on cloud to enable stable operation of smart farm for 365 days. In order to solve the difficulties of users of smart farm, the IoF cloud collects, stores and analyzes agricultural big data based on public cloud and supports farming decision making in Fig. 5 [11].

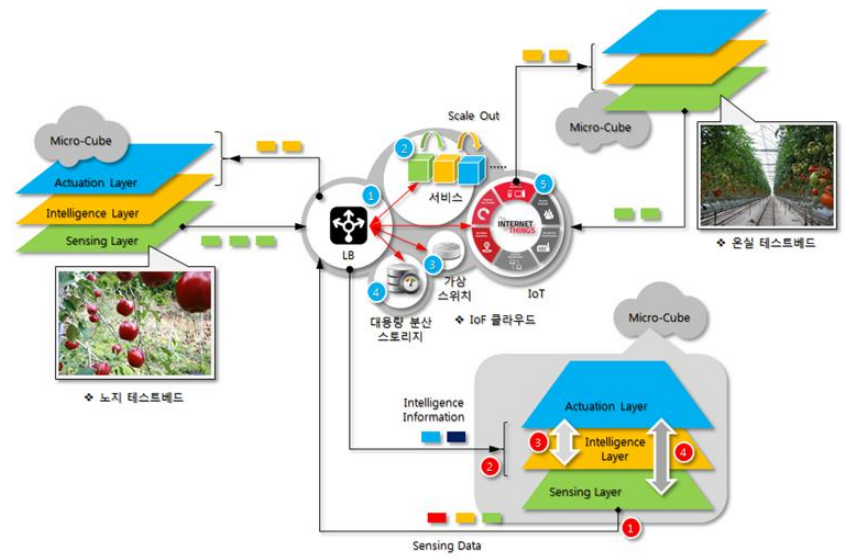

Figure 5. Concept of IoF-Cloud and Testbed of RealEnvironment

\section{IoF-based DLOTA Mechanism Design}

\section{A. IoT device-based DLOTA}

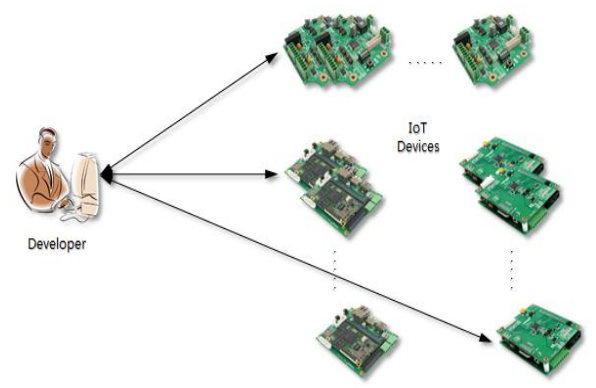

Figure 6. General Concept of SW Maintenance of IoT devices

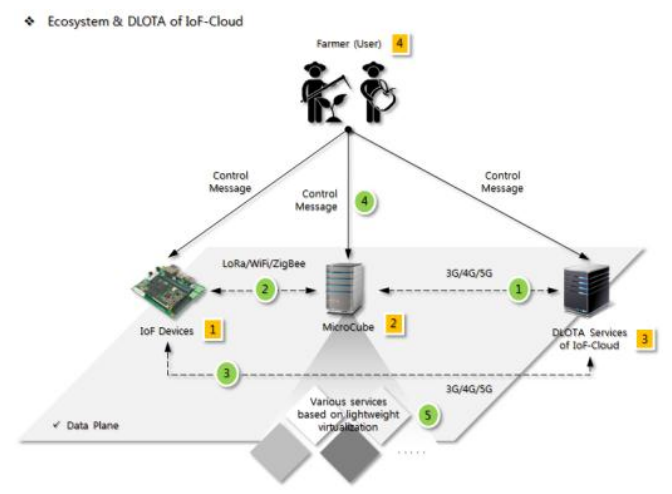

Figure 7. Diagram of DLOTA Mechanism of IoF-Cloud

As shown in Fig. 6, DLOTA of IoT or IIoT proposed in this paper can easily solve complex problems of installing and upgrading all types of IoT devices one by one and can be applied to various areas. Fig. 7 shows the environmental 
diagram of the DLOTA mechanism of the proposed IoT-based IoF-Cloud.

\section{B. Functional architecture and DD/MO of IoT-based DLOTA Mechanism}

The DLOTA mechanism based on IoT is operated by the Download Description (DD) and MO (Media Object) information between Download Agent and Server similar to DLOTA of OMA. DD is composed of elements for describing MO, and DD. Fig. 8 shows the functional architecture of the IOT-based DLOTA mechanism.

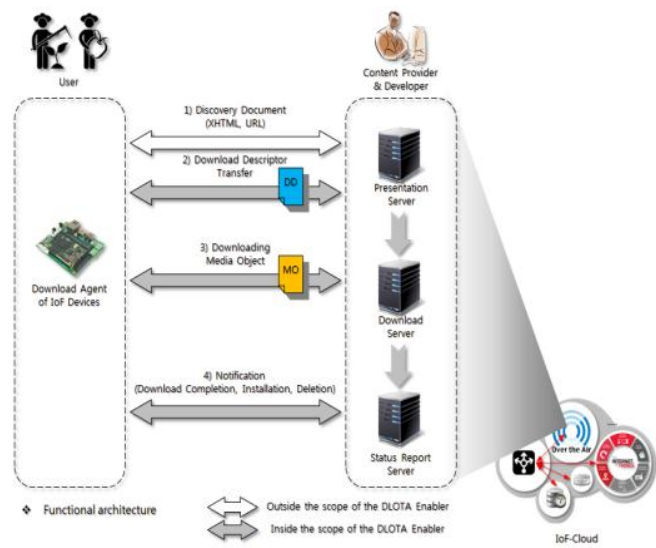

Figure 8. Functional Architecture of IoT-based DLOTA Mechanism

Content Provide \& Developer will configure cloud resource (IoF-Cloud) with server virtualization technology such as Present Server, Download Server, Status Report Server, and so on. In particular, Ubuntu Linux supports all types of containers (process container Docker, machine container LXD, and application container Snapd). The user receives the DD from the Present Server, receives the MO from the Download Server, and sends the status information to the Status Report Server when downloading and installation is completed. Fig. 9 shows a diagram of a cloud-based DLOTA service design to support IoT. The discovery application in IoT device and IoT gateway (MicroCube) download DD from present server which is virtual machine of cloud and analyze contents (refer to (1) in Fig. 9]), and DD includes CapBAC access control information.

IoT Gateway obtains MO information for IoT devices by DD information and installs it in Download User Agent module (refer to (2) in Fig. 9]). The Download User Agent will check the MO and CapBAC access control (refer to (3) in Fig. 9]). If there is no problem in the normal situation and the CapBAC access control does not occur, the cloud-based OTA service To search for and download the corresponding MO (refer to (4) in Fig. 9]). The end user's authorization (refer to (5) in Fig. 9) gives the download permission and the downloaded MO is stored in the storage of the IoT Gateway (refer to (6) in Fig. 9). The IoT Gateway performs the storage cache function for IoT devices and also converts the global traffic of 3G / 4G / 5G to local traffic such as LPWAN (Low Power Wide Area Network).

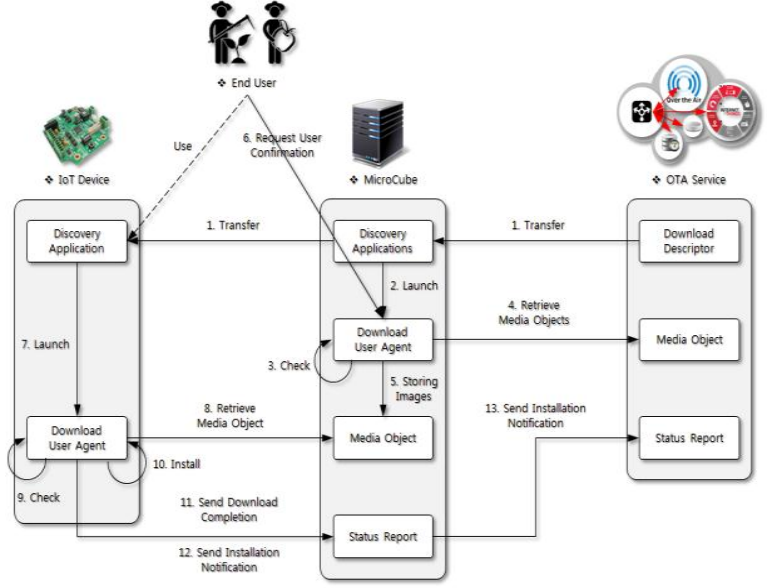

Figure 9. Service Design of Cloud-based DLOTA

The end-user IoT device acquires MO information corresponding to its type and installs it in the Download User Agent module (refer to (7) in Fig. 9). The Download User Agent will search for and download the MO from the IoT Gateway (refer to (8) in Fig. 9). Download User Agent will check and install MO by information and CapBAC access control (refer to (9) and (10) in Fig. 9). When the MO is installed in the IoT device, the status information of the download and installation completion is transmitted to the IoT gateway (refer to (11) and (12) in Fig. 9). The status and history information of the IoT device is managed by this information. In addition, the IoT Gateway will send status information summarized to the Status Report Server of the cloud-based OTA service (refer to 13)in Fig. 9).

\section{Basic of DLOTA}

The basic DLOTA procedure is shown in Fig. 10, and the DD and MO contents are found by the Discovery Application (refer to (1) in Fig. 10]). At this time, the IoT device requests DD from the IoT gateway (refer to (2) in Fig. 10), and the IoT gateway transmits the requested DD (refer to (3) in Fig. 10). The IoT device checks the MO information to be downloaded by the DD transmitted from the IoT Gateway and the Capability of the IoT device, and the CapBAC access control proceeds (refer to (4) in Fig. 10]). The IoT device will ask the user for approval and will be authorized to download the MO by user's approval (refer to (5) and (6) in Fig. 10). When the IoT device requests the MO from the IoT gateway, the IoT gateway transmits the requested $\mathrm{MO}$ (see (7) and (8) in [Figure 11]). When the transfer of the MO is completed, the IoT device uses the MO to install the firmware and software (see (9) in Fig. 10]) and sends the installed status information to the IoT Gateway at the completion of the installation (refer to (10) in Fig. 10]). 
Proc. of the Sixth Intl. Conf. Advances in Computing, Communication and Information Technology- CCIT 2018

Copyright (C) Institute of Research Engineers and Doctors, USA. All rights reserved.

ISBN: 978-1-63248-149-8 doi: 10.15224/978-1-63248-149-8-11

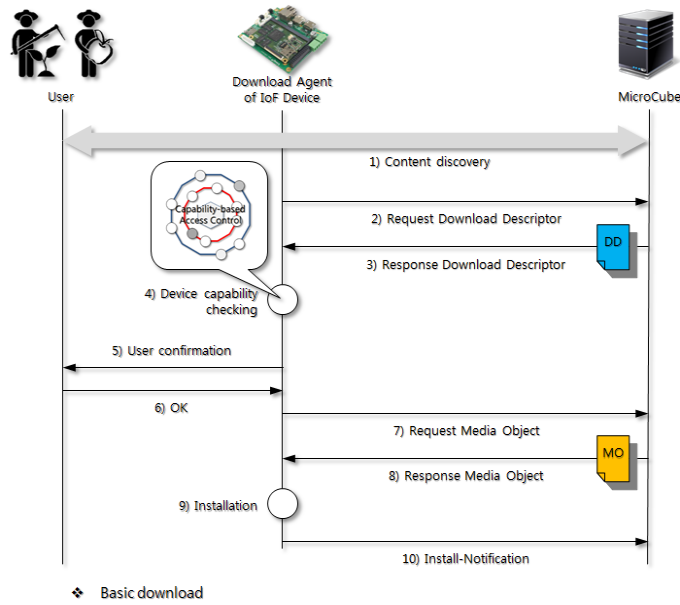

Figure 10. Basic Process of DLOTA

\section{Update and Delete of Media Object}

The procedure and procedure for updating the existing firmware or software to the latest version are shown in Fig. 11 and Fig. 12, and the latest version of MO information is obtained by the Discovery Application than the version information of the existing MO.

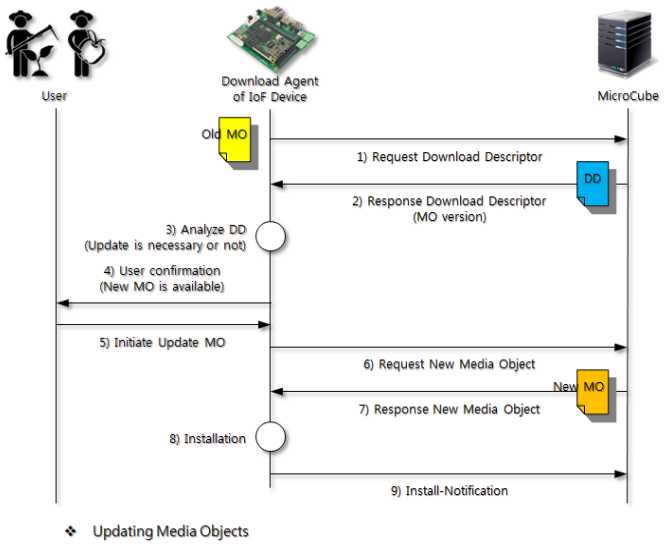

Figure 11. Update Process of Media Object

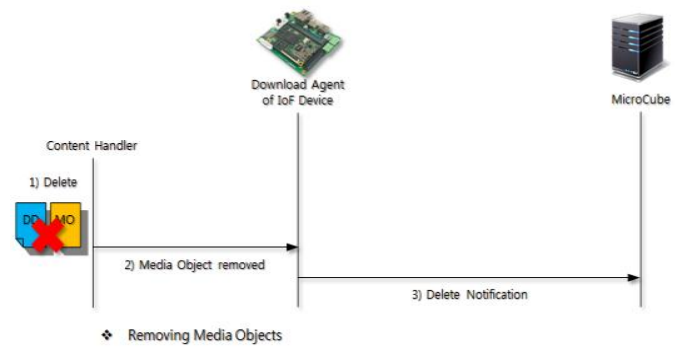

Figure 12. Remove Process of Media Object

The IoT device requests the latest version of the DD information to the IoT gateway, and the IoT gateway transmits the requested DD to the IoT device (refer to (1) and (2) in Fig. 11). The transmitted DD is analyzed to check the firmware and software for update and capability (refer to (3) in Fig. 11). If the update of the firmware or software by the version information and the capability is valid, the end user is requested to approve and authorized by the end user (refer to (4) and (5) in Fig. 11). The IoT device requests the new version of MO from the IoT gateway, and the IoT gateway transmits the requested MO (refer to (6) and (7) in Fig. 11). The IoT device performs the installation process using the downloaded MO (refer to (8) in Fig. 11) and sends the status information to the IoT gateway at the completion of installation (refer to (9) in Fig. 11). Fig. 12 shows the process of removing the existing MO. The content handler of IoT device removes the existing DD and MO (refer to (1) and (2) in Fig. 12]) as new firmware or software is installed, The IoT device informs the IoT Gateway of the status information of the existing MO removed (refer to (3) in Fig. 12).

\section{Conclusion}

The heart of IoT gives new utility and value with connectivity among things around people to the human. In future, Industrial environment will be intimately connect all among machines and machines or factories and factories in all processing, and by digitizing of all goods and production lifecycle, which is a combination of virtual world and real world, the digital factory will become reality eventually. The proposed IoT or IIoT-based Download OTA (Over-the-Air) provides a flexible mechanism for downloading media objects of any format and size over the network. In this paper, DLOTA for IOT is designed and constructed based on OMA OTA, and DLOTA, media object update and deletion procedure, DLOTA based on broadcast protocol are designed in detail respectively. In addition, the DLOTA provides lightweight encryption and partial security by CapBAC technology.

\section{Acknowledgement}

This research was supported by Basic Science Research Program through the National Research Foundation of Korea(NRF) funded by the Ministry of Science, ICT and Future

\section{References}

[1] ConnectingLab, "IoT: A huge link that goes beyond the cloud and big data," Future window Press, 2014.

[2] SeokHee Han, HeyoungSik Choo, DaeSoon Hong, "The Fourth Industrial Revolution to Determine the Future - Industry 4.0," Paperroad, 2015.

[3] Industrial Intermnet Consortium, "Industrial Internet of Things Volume G4: Security Framework," 2016.

[4] OMA, "Download Over the Air Specification," Sep., 2006.

[5] OMA, "Download Over the Air Architecture," Aug, 2006.

[6] Roger Hampel, "Keeping the connected car current with SOTA/FOTA," Automative Linux Summit, Sep. 19, 2012. [Internet] 
https://events.linuxfoundation.org/images/stories/pdf/als2012_hampel.pd $\mathrm{f}$

[7] Ed Tittel, "CISSP: Certied Information Systems Security Professional Study Guide," InfoPub, 2004.

[8] Sergio Gusmeroli, Salvatore Piccione, Domenico Rotondi, "A capability-based security approach to manage access control in the Internet of Things, " Mathematical and Computer Modeling 58, Elsevier, 2013, p.1189-1205.

[9] Jose L. Hernandez-Ramos, Antonio J. Jara, Leandro Mariın, and Antonio F. Skarmeta, "Distributed Capability-based Access Control for the Internet of Things," Journal of Internet Services and Information Security (JISIS), Vol. 3, no3/4, 2013, p.1-16.

[10] Osami Ogasawara, "Makers Evolution, “ The Forest, May 2016.

[11] ByungRae Cha, MyeongSoo Choi, BongKook Kim, OhSeung Cheon, TaeHo Han, JongWon Kim, Sun Park, "Research of Next Generation IoF-Cloud based Smart Geenhouse \& Services," Korea Smart Media Journal, Vol.5, No.3, Sep., 2016, p.17-24.

ByungRae Cha:

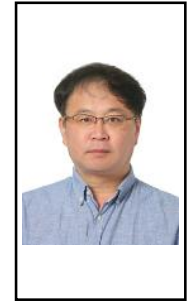

ByungRae Cha is a research professor at School of Electrical Engineering and Computer Science and Super Computing \& Collaboration Environment Technology (SCENT) Center, GIST, Korea. He received the Ph.D. degree in Computer Engineering from National Mokpo University in 2004 and the M.S. degree in Computer Engineering from Honam University in 1997. Prior to becoming a research professor at GIST, he has worked as a research professor in Dept. of Information and Communication Eng., Chosun University, and professor in Dept. of Computer Engineering, Honam University, Korea. His research interests include Computer Security of IDS and P2P, Machine Learning, and Cloud Computing.

Sun Park:

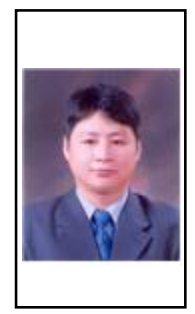

Sun Park received the B.S. degree in Computer Engineering from Jeonju University in 1996. He received the M.S. degree in Information \& Communication Engineering from Hannam University in 2001, and the Ph.D. degree in Computer \& Information Engineering from Inha University in 2007. From November 2013, he has working the School of Electrical Engineering and Computer Science, Gwangju Institute of Science \& Technology (Gwangju, Korea), where he is a research professor. Prior to becoming a researcher at GIST, he has worked as a research professor at Mokpo National University, a postdoctoral at Chonbuk National University, and professor in Dept. of Computer Engineering, Honam University, Korea. His research interests include Data Mining, Information Retrieval, Information Summarization, Convergent Marine ICT, Smart Farm, and IoT-Cloud computing.
JongWon Kim:

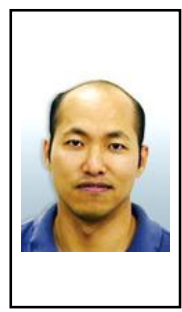

JongWon Kim received the B.S., M.S. and Ph.D. degrees from Seoul National University, Seoul, Korea, in 1987, 1989 and 1994, respectively, all in control and instrumentation engineering. In 1994-1999, he was with the Department of Electronics Engineering at the Kongju National University, Kongju, Korea, as an Assistant Professor. From 1997 to 2001, he was visiting the Signal and Image Processing Institute (SIPI) of Electrical Engineering Systems Department at the University of Southern California, Los Angeles, CA. USA, where he has served as a Research Assistant Professor since Dec. 1998. From September 2001, he has joined as an Associate Prof. at School of Electrical Engineering and Computer Science, Gwangju Institute of Science and Technology (GIST, formerly known as K-JIST), Gwangju, Korea, where he is now serving as a Professor. He is focusing on networked media systems and protocols including multimedia signal processing and communications. Dr. Kim is a senior member of IEEE, a member of ACM, SPIE, KICS, IEEK, KIISE, and KIPS.

Byeong-Chun Shin:

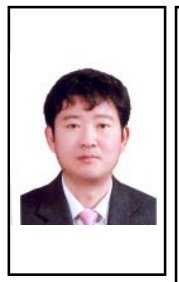

Byeong-Chun Shin received the B.S., M.S. and Ph.D. degrees at department of Mathematics from Kyungpook National University, Daegu, Korea, in 1986, 1988 and 1996, respectively. He visited Purdue University as a post doctor in 2000 and he was a research assistant professor in Ajou University. From September 2001, he had served as a research assistant professor in Seoul National University and then from August 2002, he has joined as an assistant professor at Department of Mathematics, Chonnam National University, Gwangju, Korea. In 2005, he visited the National Center of Atmospheric Research (NCAR), Colorado, USA as a Scientific Visitor. He is now serving as a Professor from October 2011. His main research is focusing on the numerical analysis and methods including the Artificial Neural Network and Deep Learning with Computer Vision. 